\title{
Collecting responses through Web page drag and drop
}

\author{
M. ANNE BRITT \\ Northern Illinois University, Dekalb, Illinois \\ and \\ GARETH GABRYS \\ Apropos Technology, Inc., Oakbrook Terrace, Illinois
}

\begin{abstract}
This article describes how to collect responses from experimental participants using drag and drop on a Web page. In particular, we describe how drag and drop can be used in a text search task in which participants read a text and then locate and categorize certain elements of the text (e.g., to identify the main claim of a persuasive paragraph). Using this technique, participants respond by clicking on a text segment and dragging it to a screen field or icon. We have successfully used this technique in both the argument element identification experiment that we describe here and a tutoring system that we created to teach students to identify source characteristics while reading historical texts (Britt, Perfetti, Van Dyke, \& Gabrys, 2000). The implementation described here exploits the capability of recent versions of Microsoft's Internet Explorer Web browser to handle embedded XML documents and drag and drop events.
\end{abstract}

Over the past 5 years, there has been a substantial increase in the number of research studies conducted over the Internet (Birnbaum, 2000; Buchanan, 2000; Krantz \& Dalal, 2000; Wolfe \& Reyna, 2002). This increase is partially due to the diversity and size of the population available through the Internet, as well as to more practical issues of researchers' lab space and time requirements (Bailey, Foote, \& Throckmorton, 2000; Krantz \& Dalal, 2000; Reips, 2000). Another reason for the increase has been the advances in the power and ease of use of Web browsers and servers. Even research conducted in more controlled settings can now take advantage of the powerful scripting capabilities built into Web browsers and the ease of deploying an experiment program on a single server, rather than on multiple workstations. The latter benefit is especially important in school environments, where the file systems of individual machines are frequently locked to prevent student tampering and access to file servers may require special procedures or personnel. Our own experiences in schools (Britt \& Aglinskas, 2002; Britt, Perfetti, Van Dyke, \& Gabrys, 2000) have motivated us to look for techniques that exploit such browser-based solutions to both experimental tasks and tutorial environments. The drag and drop technique described in this article is one that enables re-

This research was supported in part by a grant to the first author from the Cognition and Student Learning program of the Institute of Education Sciences, U.S. Department of Education. Correspondence concerning this article should be addressed to M. A. Britt, Psychology Department, Northern Illinois University, Dekalb, IL 60115 (e-mail: britt@niu.edu). searchers to present a text search and identification task through a Web browser. Participants read a text and then search for answers to questions or identify particular discourse elements by clicking on the text and dragging a text unit to a screen target, where the unit can be displayed and feedback presented. The use of this technique in our research and tutorials has shown it to be both effective and enjoyable for our participants.

The use of drag and drop with text segments came out of our work on the Sourcer's Apprentice (Britt \& Gabrys, 2001; Britt et al., 2000), an application that trains students to attend to source information while reading historical documents. Students using the Sourcer's Apprentice read short documents and filled in on-screen notecards by dragging phrases from the documents to screen icons. When the students dropped a response, it was automatically scored and then entered into a notecard. The participants generally found this to be an enjoyable activity, and studies of their use of the Sourcer's Apprentice (Britt \& Aglinskas, 2002) showed it to be an effective learning activity.

Drag and drop is particularly well suited to search-andcategorize tasks in which participants view some number of screen items that need to be classified into multiple categories. It is possible to present the items as a list and repeat the response categories for each list item or to present the items freely and place input boxes by each category choice, into which the participants can type their responses (see Figure 1), but these methods either break up the continuity of the items and impose an order on the task or make automatic checking difficult because of the problem of dealing with free responses. Drag and 


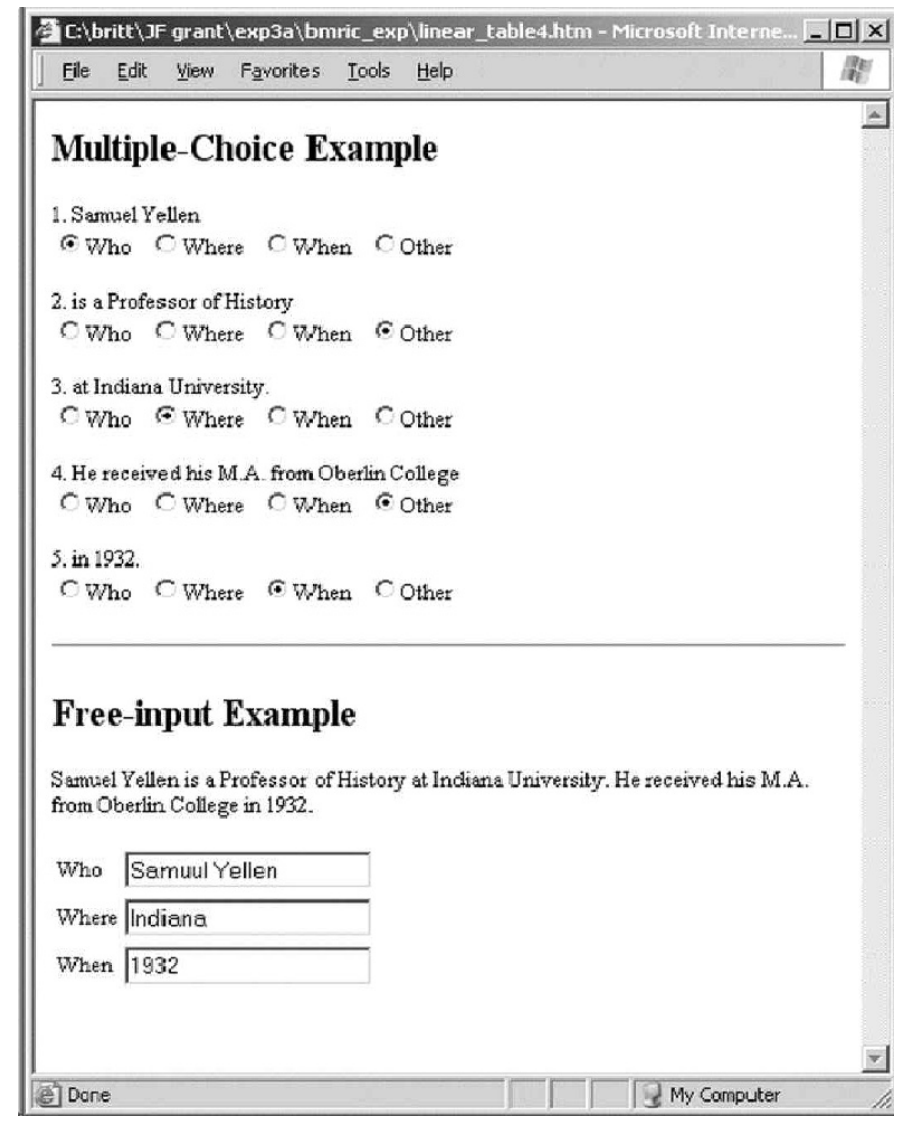

Figure 1. Example of interface for presenting a multiple response task as multiple choice and free input.

drop enables items to be displayed naturally, and it enables automatic checking and immediate feedback, because the response is restricted (see Figure 2). It is also a fairly natural way to respond: The specific item is "picked up" and "placed" on the desired response.

In this article, we describe a technique for collecting data, using drag and drop on a Web page. We also briefly describe how we used this technique in a research study in which students' ability to identify argument elements in a persuasive essay was examined.

\section{Web Page Drag and Drop of Text Segments}

The technique we have developed takes advantage of two features of Version 5 of Microsoft's Internet Explorer (IE) Web browser ${ }^{1}$ —namely, XML data islands and drag and drop event handling.

XML documents and data islands. XML (extensible markup language) is a standard for specifying userdefined commands and data that can be sent as text files through Web servers, to be processed by some application. It has a syntax similar to that of the HTML language used to define Web pages, but the bracketed elements are defined by the user, rather than by a standards committee. The value of XML is that it defines a systematic format for specifying structured data. For exam- ple, consider the XML document in Table 1. This XML document specifies a Book element that contains information about a book, such as a title, an author name, publisher information, and an "about the author" page. ${ }^{2}$ As in HTML, XML elements enclose information with begin and end tags, and they can have attributes. The Book element, for example, specifies an id attribute. This id attribute is arbitrary and is simply a useful way to uniquely identify the Book. The other embedded elements provide specific information about the document's author and publisher, and the Page element specifies the content of the "about the author" page. Keep in mind that this document and its elements are completely arbitrary; we could have used different labels, such as "Stimulus" for "Book," "Passage" for "Page," and so forth. All that is required is agreement among the users of the document format.

Although XML resembles HTML, it is syntactically more strict. First, all the elements must have matching opening (e.g., $<$ Book $>$ ) and closing (e.g., $</$ Book $>$ ) tags. Second, XML is case sensitive, so "Book" is not the same as "book." Third, the values of all attributes must be quoted (e.g., rank="1"). Fourth, when elements contain any text that can be interpreted as markup, such as the characters: $<,>,-, \&$, and so forth, the section 


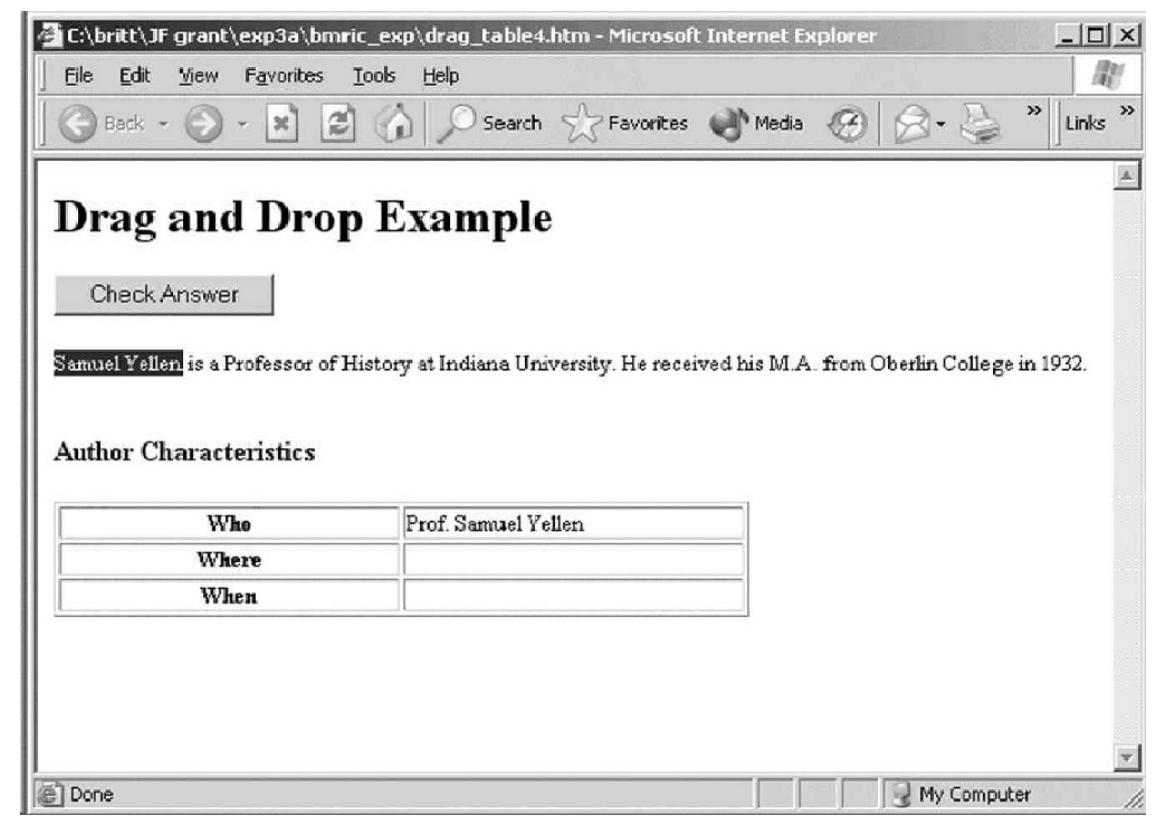

Figure 2. Example of interface for presenting a multiple response task, using drag and drop.

must be enclosed within the special CDATA element, delimited by brackets (see the example in Table 1). The CDATA element instructs the XML parser to ignore any markup characters contained within it.

Normally, XML documents are sent as stand-alone files from a client to a Web server for processing, or they may be returned from a Web server and displayed using templates written in a special style language (XSL). However, an interesting new way to use XML documents is to insert them into Web pages as data islands. IE Versions 5 and later support a new HTML tag that enables the page's author to embed and reference an XML document within a Web page. To embed our example XML document in a Web page, we have enclosed it within the new $<$ XML $></$ XML $>$ tags. Once inserted into a Web page, the data island will be automatically parsed when the page is loaded into the client's browser, but it is not displayed. Scripts embedded in the page can then access this structured data, using a standard set of built-in functions (known collectively as the document object model, or DOM). For example, loading a Web page that contains the data island from Table 1 and the script in Table $2^{3}$ will display a button that, if pressed, will cause a message box to pop up, displaying the text of the first Answer element from Table 1.

The JavaScript ${ }^{4}$ code in Table 2 defines a function called checkAnswer that performs a simple hierarchical lookup of information in the data island. "MyDocument" refers to the HTML element that contains the XML document. "XMLDocument" references the root node of the hierarchy. Each node contains a collection called childNodes. The root node has a collection of one child node that corresponds to our Book element. The checkAnswer function accesses the root node and as- signs the root nodes's child to the bookNode variable. It then calls a DOM function called getElementsByTagName, which searches for all elements named Question and returns a collection of all matches. The script iterates through each question until it finds one whose id attribute matches the one passed into the function ("WhoDrop"). It then iterates through all the child nodes of the question until it finds one whose id matches the passedin answer id ("auth_dt1"). If it finds the answer, it returns the text associated with it ("Prof. Samuel Yellen"). The actual text of the answer is contained in a text node child of the Answer element and is accessed via the nodeValue property. If a matching answer is not found, the function returns a null string. The script code at the bottom of Table 2 calls the checkAnswer function and pops up an alert box with the result. Note that if the XML document contains any syntax errors, the root node will be null.

A drag and drop document. Our drag and drop technique exploits data islands in order to create a text that contains uniquely identifiable text segments, along with possible response answers. Examining the Page element of the XML document in Table 1 shows that the contents of the page have been broken up into uniquely identifiable units. The HTML SPAN element provides a way to do this. A SPAN element has no default effect on the way the browser displays the text it encloses. By adding an id attribute to the SPAN, we can uniquely identify a text segment. SPAN elements can also be assigned styles that can be used to highlight the text unit, change its color, or alter its appearance in some other way. The CDATA section wrapped around these SPAN elements enables HTML to be embedded within the contents of the XML Page element without having the XML parser try to in- 
Table 1

An XML Document Embedded as a Data Island in a Web Page

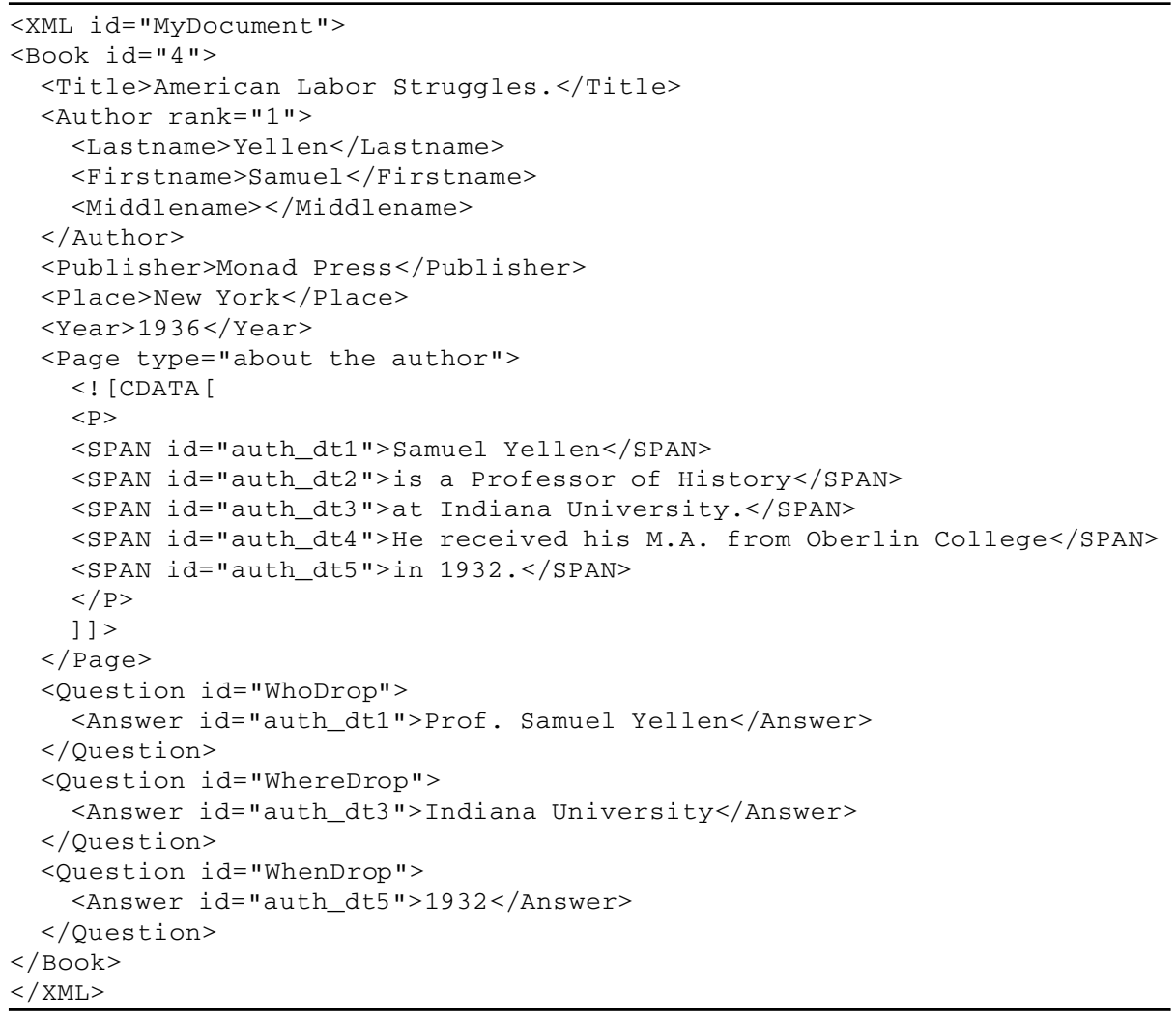

Note-The source code is available at http://www3.niu.edu/psychology/britt.

terpret it. We can later extract this content and assign it to a displayable HTML control.

The document in Table 1 breaks the Page text into five uniquely identified SPAN elements. The SPAN's identifier is used to associate each text segment with a response to our drag and drop operation. The bottom part of Table 1 defines Question and Answer elements. Question elements correspond to questions. These elements have an id attribute that we will use to associate each Question with a visible HTML object that will serve as a drop target. Contained within each Question is one or more Answer elements. Each Answer element has an id that corresponds to the ids of the SPANs in the document's Page element. In the example, there is one Answer element for each correct answer to the corresponding question. We assume that any responses not contained as answers are incorrect.

Drag and drop handling in Internet Explorer. IE Versions 5 and later define special events and objects to handle drag and drop operations. To enable drag and drop operations, the Web page must define script functions to handle these events and associate the functions with HTML objects. Tables 3 and 4 show how this works. If the contents of Table 3 are added to those of Tables 1 and 2, we get a Web page that looks like Figure 2. The code in Table 3 defines an HTML SPAN element named theDoc and provides some JavaScript that looks up the contents of the XML document's Page elements and assigns it to the theDoc element. Note that this SPAN element also associates the handlers myMouseDown and myDragStart to the onmousedown and ondragstart events. The onmousedown event is triggered when the user clicks anywhere in the text. The ondragstart event is triggered when the user holds the mouse button down and begins to move the mouse pointer.

The code in Table 3 next defines an HTML table. This table contains three rows for each of three author characteristics. The first column of these rows provide the name of the characteristic. The second column contains a space character to force the cell borders to draw when the cell is empty. The TD elements for the second column have an id attribute whose names correspond to the Question elements of our XML document. They also associate the my Drag Over function to the ondragenter and ondragover events. The Table element itself associates the myDrop function with the ondrop event. The ondragenter event is triggered when the user moves the mouse pointer into the cell while dragging. While the mouse pointer is inside the cell, the ondragover event is triggered whenever the mouse moves until it leaves the cell. The ondrop event is triggered when the user releases the mouse button while inside the table.

The code for the handler functions is shown in Table $4 .{ }^{5}$ The first handler is myMouseDown, which handles the 
Table 2

Script That Retrieves a Question Answer From an XML Data Island

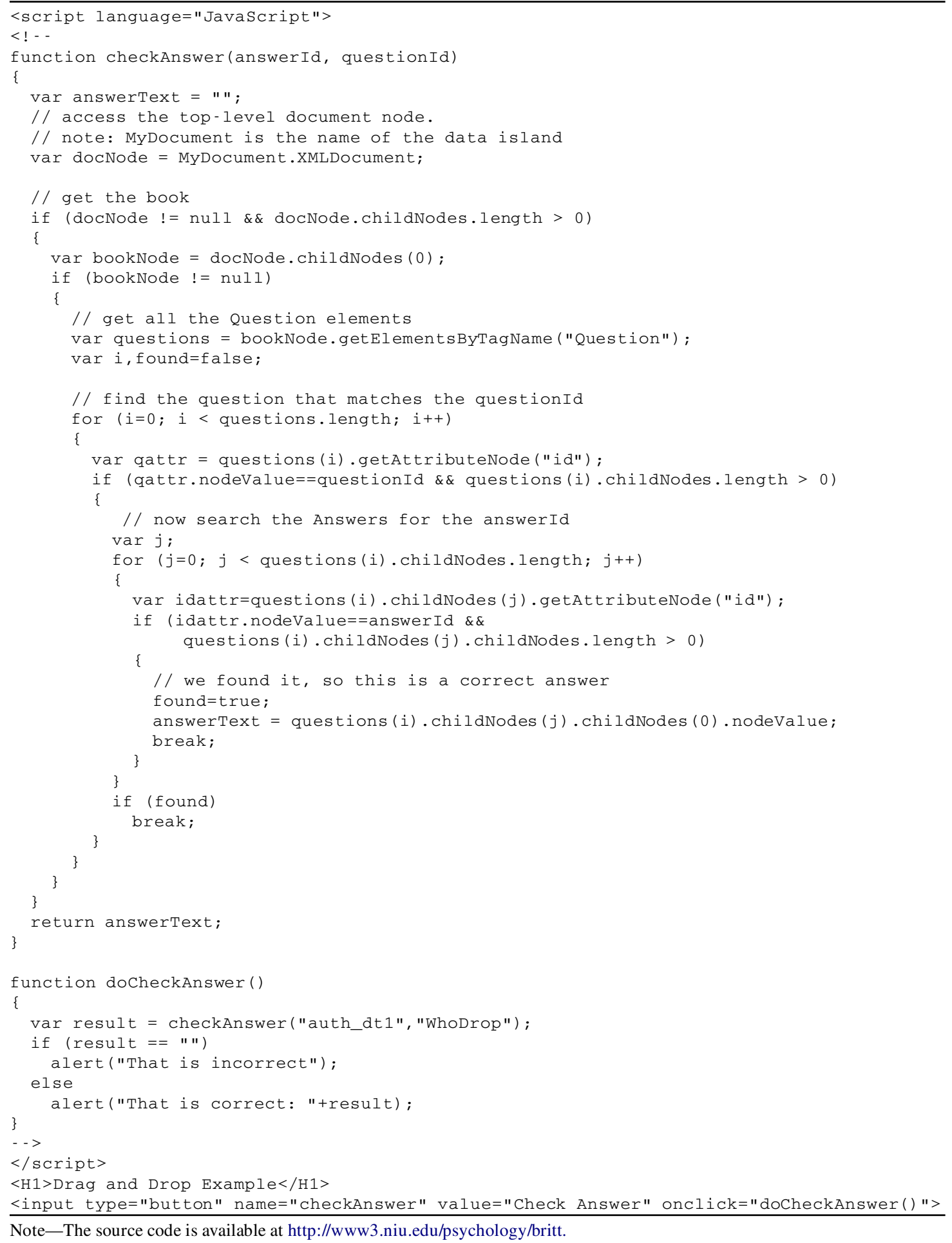

onmousedown event. This function selects the text in the SPAN element where the mouse was clicked. The next handler, myDragStart, handles the ondragstart event by copying the id of the selected SPAN element to the system- defined dataTransfer object and setting the event object's eventAllowed property to "copy," to set the drag cursor appearance. Next comes the myDragOver function, which handles both the ondragenter and ondragover 
Table 3

HTML and Script Code to Display the Data Island Content and a Table of Drop Targets

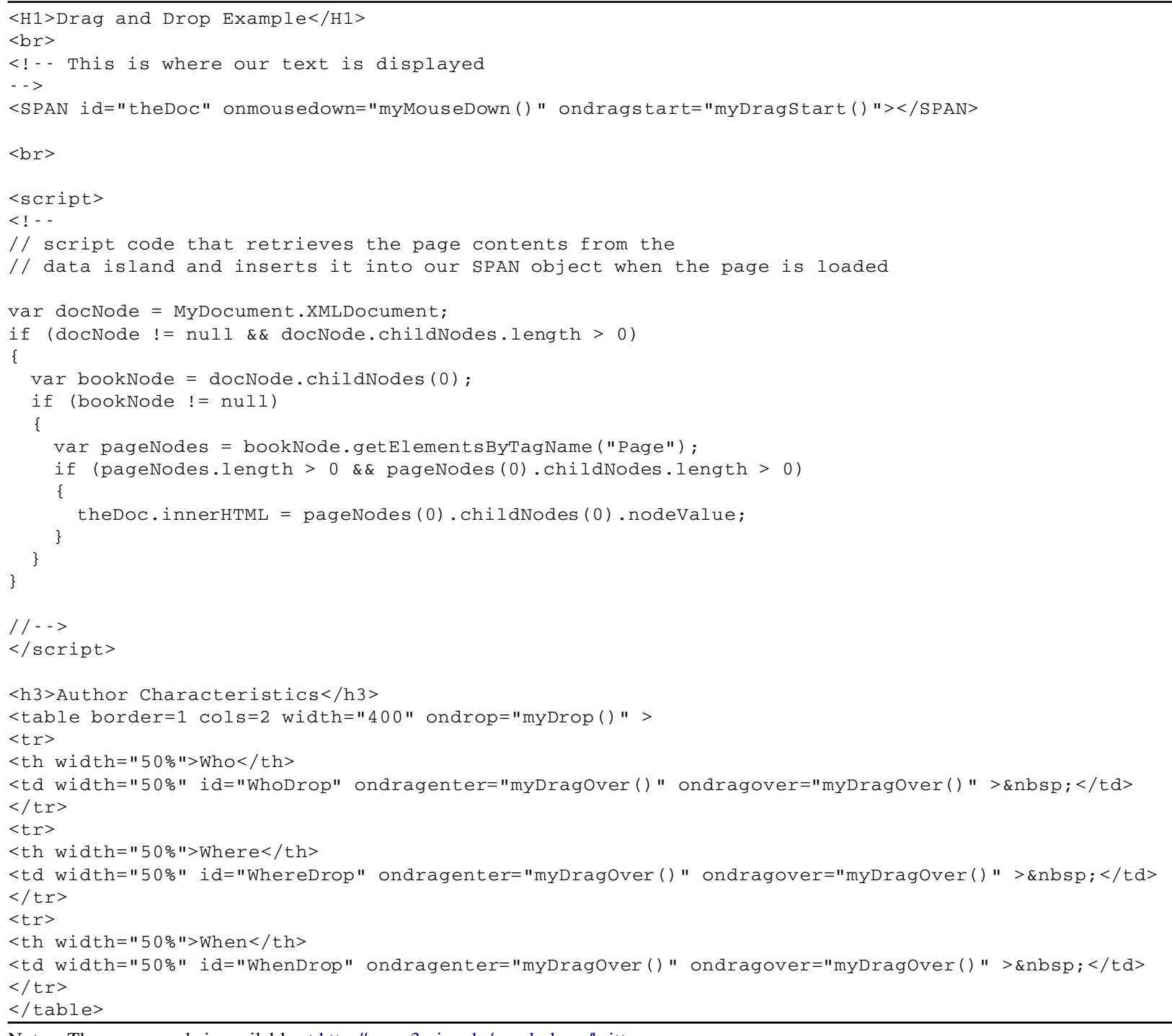

Note-The source code is available at http://www3.niu.edu/psychology/britt.

events. This handler enables the table cell to function as a drop target by setting the dataTransfer object's dropEffect property to "copy" and setting the function's returnValue to false so that the default handler is not called. The final handler is myDrop, which handles the table's ondrop event. This function retrieves the id of the dragged SPAN element from the dataTransfer object and the id of the cell where the drop occurred from the event object's srcElement property. It then calls the checkAnswer function and displays the results. If the result is correct, it also inserts the answer text into the cell where the drop occurred.

It is important to note that the technique we have described takes place completely on the client side (i.e., the user's browser). For obvious security reasons, Web pages cannot write responses to files on the client. Instead, the response must be sent back to the server and recorded there. The Appendices contain a complete example that collects event times, posts the response and response times to the server, and $\log s$ the data to a file on the server. We will describe in general terms how this works in the next section.

Recording data. The most common method for transferring response data from the client to the Web server is through a form submission. Forms are HTML elements that name and encapsulate data transferred from a Web browser to a server. Although forms are most commonly used to display controls, such as drop lists and edit boxes, there are also hidden form elements that can be populated and submitted from scripts. When a user makes a drag and drop response, the script writer has the option of immediately filling in a form element and submitting the data to the server or of collecting the results of several drops and submitting all the results at 
Table 4

Drag and Drop Event Handlers

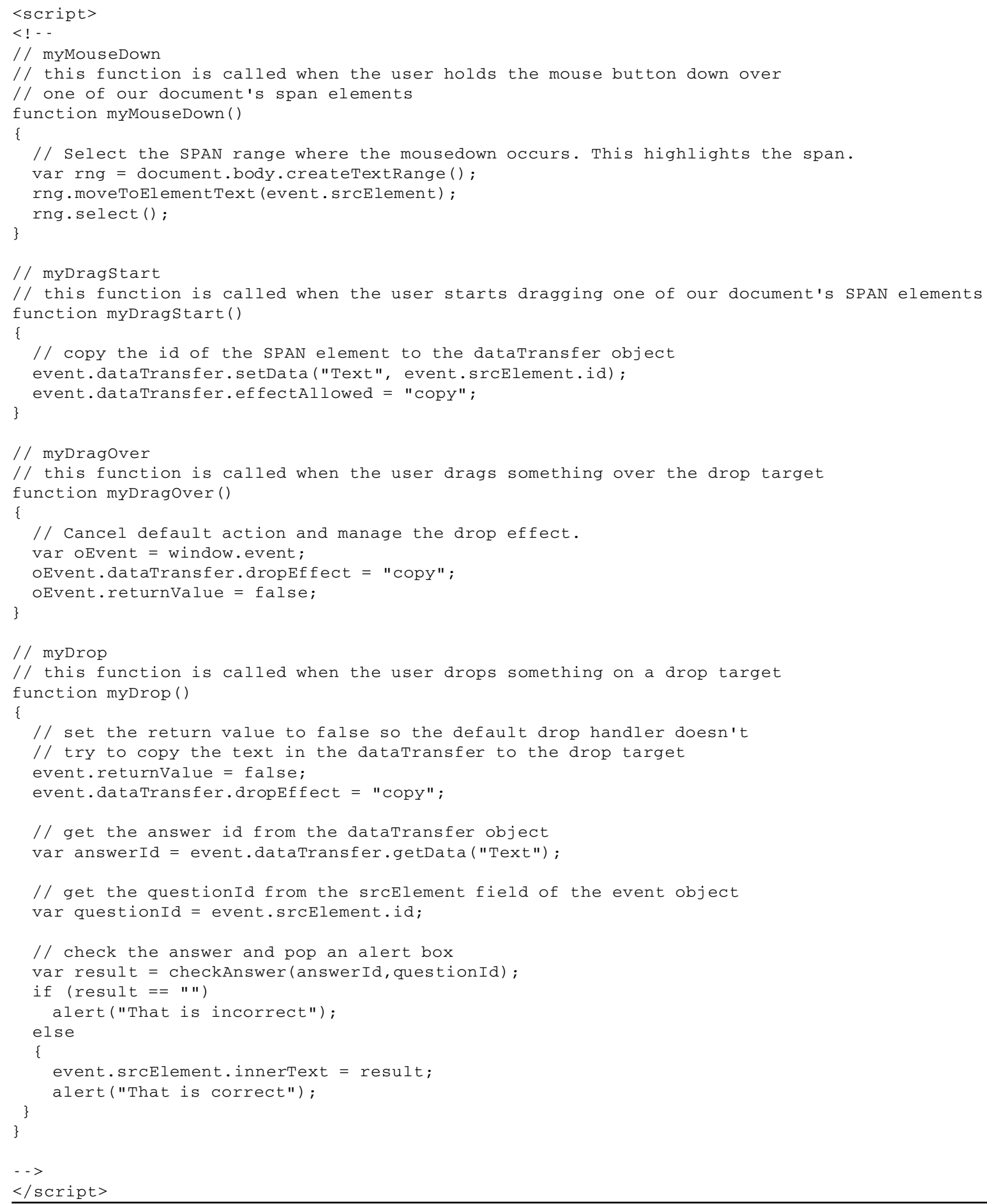

once. One important consideration is that form submission causes the browser window to refresh. This may be perceived as an interruption, especially when the page renders slowly because of its size or the client's slow connection to the server. Sometimes, however, submissions after each drop are necessary, as in the case in which the response requires extensive analysis on the server. 
The complete example, given in Appendix A, ${ }^{6}$ performs a form submission after every drop. An HTML form element named dropForm has been added to the page. This form contains hidden input elements for returning the document identifier, the id and contents of the dropped text unit, and the time of the drop operation on the client. The ondrop handler (myServerDrop) calls fillFormAndSubmit to insert values into the hidden form elements and then submit the form. The submission must now be unpacked and recorded by the server. There are several server-side technologies for handling Web form submissions (Schmidt, 2000), such as CGI, ASP, ISAPI, and JSPs. Our example (in Appendix B) ${ }^{7}$ uses an Active Server Page (ASP) page written in VBScript to extract the data from the form, store it in a file, and redisplay the page. ASP pages are text files containing script code that is interpreted by Microsoft's IIS Web server. CGI scripts (usually written in Perl) are the more common choice on Unix-based servers, such as Apache. Alternatively, a Java Server Page (JSP) server, such as the opensource Tomcat, may be used on both platforms.

Timing. Our example scripts and the experiment that follows record the time stamp of the drag and drop events. We collect these time stamps on the client and transfer them to the server because the client time, although subject to the vagaries of the browser and operating system, is still a better estimate than the time on the server, which is subject to network speed and congestion and server load. Because we are interested only in the duration between two time stamps, it is not important that time might differ among clients. It should be kept in mind, however, that the accuracy of timing with this or any browser-based application is limited to seconds or, at best, tenths of seconds.

\section{AN EXPERIMENT USING DRAG AND DROP DATA COLLECTION}

We have recently used the technique we have described to collect data for a study on argument comprehension. The participants read paragraph-long arguments and were asked to identify the main claim, reasons, backings (evidence), and counterreasons. They entered their responses by clicking on a phrase from the argument and then dragging and dropping it into a cell of a table labeled with the appropriate argument element (see Figure 3). Because of the length of the arguments, the drop table was presented in a separate frame below the passage, enabling the passage to be independently scrolled. The passage identifier, the dropTarget (table cell), the dropped element, and time of the drop were submitted to the server on each drop and recorded in a file. When the page refreshed after the submission, the dropped text was displayed in the table cell where the element was dropped. No feedback was provided. When the participants considered themselves finished with a passage, they clicked a button below the drop table to move on to the next passage.

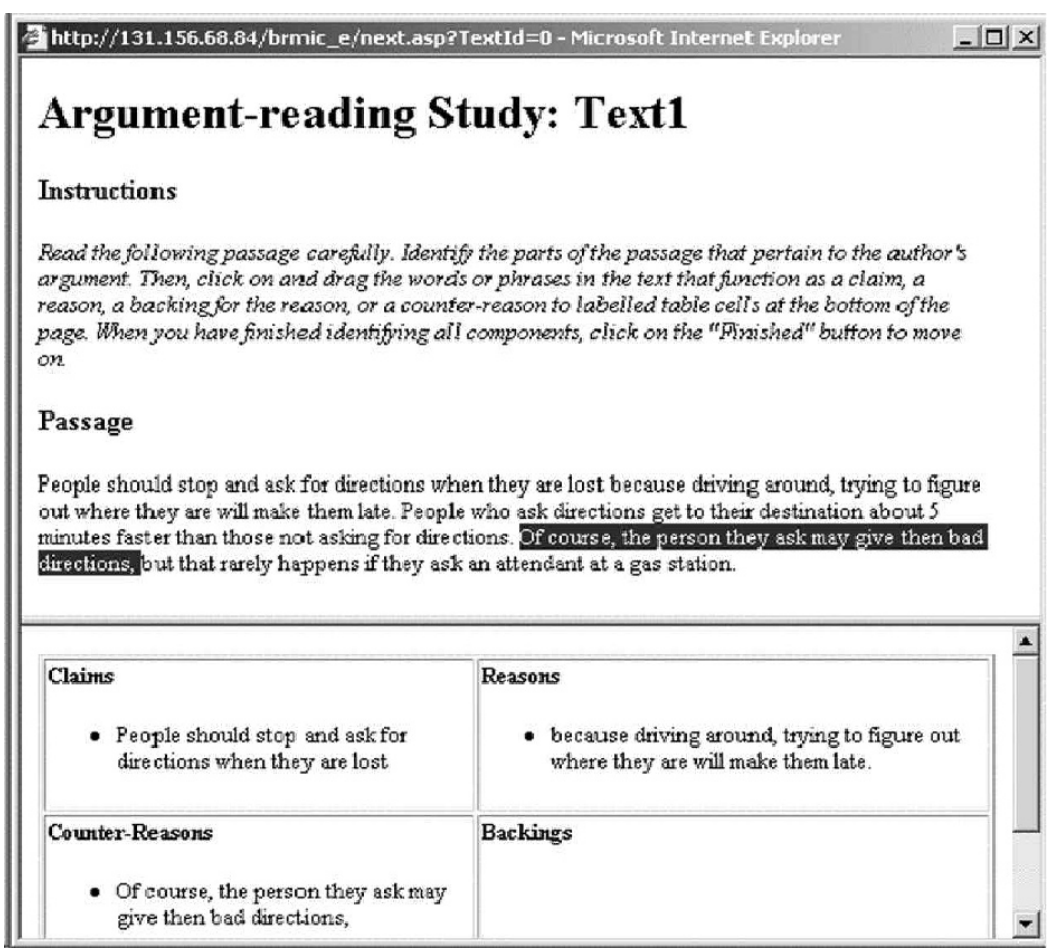

Figure 3. Screen capture from the experiment demonstrating the drag and drop procedure for an argument element classification task. 


\section{Method}

Participants. Twelve students from a psychology course on research methods participated in this experiment for course credit.

Materials. Two practice and six experimental arguments were presented to the participants, using a Web browser. Each argument consisted of at least a main claim and one reason. The arguments varied in length from 33 to 630 words. On average, the passages contained 1.33 claim statements, 4.67 reason statements, 1.5 counterreasons statements, 6.50 backing statements, and 4.33 statements of other types. Each experimental argument had two versions: implicit and explicit. The explicit arguments added clear cues to signal the presence of each argument element, such as "We propose that ..." and "The first reason is ..."

Design and Procedure. The participants were randomly assigned to one of two cue conditions, explicit or implicit. The participants read one screen page of instructions describing each argument component. Then they were given an example argument that contained one of each argument component, along with the correct classification of each text element that corresponded to one of the components. Finally, they were given instructions on using the drag and drop procedure. All the participants were familiar with drag and drop, and no one required additional assistance. They were told that they could fill the boxes in any order and were given a practice argument. The participants completed the eight arguments and then were given an opportunity to critique the interface.

\section{Results and Discussion}

The focus of the Results presentation is on the type and nature of the data logged by the server. Table 5 shows 1 participant's data file output. This output indicates the time the server logged the item, the ASP session id, the argument number (5), the drop location (claim, reason, backing, or counterreason), the type of argument (policy), the element dropped ( 1 1), whether the drop was correct or incorrect (crt), the drag start time, and the drop time.

The proportion of each type of argument element correctly answered is shown in Table 6 . The participants were able to correctly identify claims with $70 \%$ accuracy but had more difficulty with the other components (40\%, $52 \%$, and $33 \%$ accuracy for the reasons, counterreasons, and backing, respectively). Table 7 presents the average latency of their first drop and the percentage of first drops that were claims. The relatively short latency indicates that the participants did not read the entire passage prior to submitting their first responses. They read for an average of only $6.31 \mathrm{sec}$ before responding. The most frequent initial drop was the main claim of the argument ( $85 \%$ of the time). This provides an important insight into the participants' approach to the task. In addition to collecting information on the segment dropped into a particular box and the response time, a qualitative analysis of errors can be preformed.
Table 6

Mean Percentage of Correct Responses for Each Argument Category by Condition

\begin{tabular}{lcc}
\hline Argument Category & Explicit Argument & Implicit Argument \\
\hline Claims & 59.52 & 78.33 \\
Reasons & 35.21 & 44.82 \\
Backing & 30.16 & 35.05 \\
Counterreasons & 50.60 & 53.75 \\
\hline
\end{tabular}

To get a measure of usability, the final 7 participants evaluated the application on a 7-point scale. The average rating for overall experience was 2.71 (with 1 being wonderful and 7 being terrible). They found the application easy to use $(M=2.29)$ and mildly enjoyable $(M=3.00)$. Finally, they judged it to be a worthwhile educational experience $(M=2.00)$. When asked what they would improve, several participants recommended enabling the removal of responses from the table. In fact, some tried to drag the items in the table back to the text as a way of undoing their responses. This feature could be enabled with additional scripting.

\section{CONCLUSIONS}

This article has described a technique for collecting drag and drop responses to a text search task and has presented a small experiment in which this technique was used to learn about students' ability to comprehend written arguments. Many variations of this technique are possible: The drop target can be a table, an edit field, an icon, or any other displayable HTML object; dynamic styles and cascading style sheets (CSSs) can be used to display which text elements are dragable; and hint elements can be added to the document to provide graduated help when wrong elements are dropped. One should also look beyond the text-based nature of the task we have described. Drag and drop is more commonly used in graphical environments, and there is no reason why images could not be as easily associated with XML elements in our examples.

In general, drag and drop responding is appropriate for situations in which participants can make multiple consistent responses for text or graphic screen elements. It is also valuable for interfaces where one wants to convey a sense of "movement" or transfer of location for some element. Finally, it is useful for exploiting the semantics of the drop action-dropping a file on a folder icon might signify moving the file, whereas dropping it on a printer icon might signify printing the file. Conversely, drag and drop is not particularly well suited for

Table 5

Sample of Data Collected on the Server (Logged Time, ASP Session ID, Argument, Drop Location, Argument Type, Element Dropped, Accuracy, Drag Start Time, and Drop Time) 1/29/2003 3:27:41 PM,738327737,5,,, NewPage, ,, , 1/29/2003 3:28:26 PM,738327737,5,Claim,Tbac,policy,Drop,p1,crt,10438762819301043876283140 1/29/2003 3:28:32 PM,738327737,5,Reason,Tbac,policy,Drop,p2,incrt,1043876287480,1043876289950 1/29/2003 3:28:36 PM,738327737,6,,,,NewPage,,,, 
Table 7

Mean Response Times (in Seconds) and Percentages of Claim Responses for the First Dropped Items

\begin{tabular}{ccc}
\hline Condition & $\begin{array}{c}\text { Response } \\
\text { Time }\end{array}$ & $\begin{array}{c}\text { Percentage of } \\
\text { Claim Responses }\end{array}$ \\
\hline Explicit argument & 7.4 & 83 \\
Implicit argument & 5.2 & 86 \\
\hline
\end{tabular}

making single category responses or answering questions where the response categories frequently change. For instance, if in the experiment we conducted we had wanted the participants only to identify the reasons, it would have been much simpler to have them click on each reason. Similarly, drag and drop would be a cumbersome way to respond to a multiple-choice exam. Finally, drag and drop requires that the response item be present on the screen. For many tasks in which inferences or recall is required, it would not be possible to use this technique.

This article has also illustrated the use of XML data islands for storing complex stimulus descriptions in a Web page. Such descriptions can be embedded in an application page or stored in a database or in separate files and selected at runtime. We have used them to associate drag and drop responses and answers, but they can be used with any response method to enable client-side presentation of data or handling of responses.

Drag and drop responding and XML data islands are two new tools available to researchers and instructional designers. Now that they are available in Web browsers, they can be easily used by researchers who conduct studies with the Internet or by those who simply exploit the convenience of browser-based applications in their own lab.

\section{REFERENCES}

Bailey, R. D., Foote, W. E., \& Throckmorton, B. (2000). Human sexual behavior: A comparison of college and Internet surveys. In M. H. Birnbaum (Ed.), Psychological experiments on the Internet (pp. 141-168). San Diego: Academic Press.

Birnbaum, M. H. (2000). Decision making in the Lab and on the Web. In M. H. Birnbaum (Ed.), Psychological experiments on the Internet (pp. 3-34). San Diego: Academic Press.
BritT, M. A., \& AGLINSKAS, C. (2002). Improving student's ability to use source information. Cognition \& Instruction, 20, 485-522.

BrITT, M. A., \& GABRYS, G. (2001). Teaching advanced literacy skills for the World-Wide Web. In C. R. Wolfe (Ed.), Learning and teaching on the World Wide Web (pp. 73-90). San Diego: Academic Press.

Britt, M. A., Perfetti, C. A., Van Dyke, J., \& Gabrys, G. (2000). The Sourcer's Apprentice: A tool for document-supported history instruction. In P. Stearns, P. Seixas, \& S. Wineburg (Eds.), Knowing, teaching and learning history: National and international perspectives (pp. 437-470). New York: New York University Press.

BUCHANAN, T. (2000). Potential of the Internet for personality research. In M. H. Birnbaum (Ed.), Psychological experiments on the Internet (pp. 121-140). San Diego: Academic Press.

KRANTZ, J. H., \& DALAL, R. (2000). Validity of Web-based psychological research. In M. H. Birnbaum (Ed.), Psychological experiments on the Internet (pp. 35-60). San Diego: Academic Press.

REIPS, U.-D. (2000). The Web experiment method: Advantages, disadvantages, and solutions. In M. H. Birnbaum (Ed.), Psychological experiments on the Internet. San Diego: Academic Press.

SCHMIDT, W. C. (2000). The server side of psychology web experiments. In M. H. Birnbaum (Ed.), Psychological experiments on the Internet (pp. 285-310). San Diego: Academic Press.

Wolfe, C. R., \& ReynA, V. F. (2002). Using NetCloak to develop server-side Web-based experiments without writing CGI programs. Behavior Research Methods, Instruments, \& Computers, 34, 204-208.

\section{NOTES}

1. Although we hope that Netscape will eventually add similar features to Navigator, the reality is that the vast majority of Web surfers use Internet Explorer. According to an August 2, 2002 report from WebSideStory. com, IE is now used by $96 \%$ of users world wide. Of particular relevance to the technique described in this article, w3schools.com reports that IE Versions 5 and 6 now account for $91 \%$ of users. Even when IE is not a user's browser of choice, it is still available on all Windows-based computers.

2. Normally, the first line of an XML document is the standard header $<$ ?xml version="1.0"? $>$, which identifies the following text as an XML document and provides the XML specification version. In our example, we have dropped this header and put $<$ XML $></ X M L>$ tags around the document, because we are inserting it into a Web page.

3. The information from the combined Tables 1 and 2 is available at http://www3.niu.edu/psychology/britt.

4. Although all of the examples we present use JavaScript, VBScript can also be used to access XML data and handle drag and drop events.

5. The information from the combined Tables 1-4 is available at http://www3.niu.edu/psychology/britt.

6. The information from Appendix A is available at http://www3. niu.edu/psychology/britt.

7. The information from Appendix B is available at http://www3. niu.edu/psychology/britt.

\section{APPENDIX A}

Example of a Web Page That Can Do Both Client-Side and Server-Side Drag and Drop

Server-side drops require the server-side ASP page given in Appendix B. These pages must be run from a Microsoft IIS Server. This page is available at http://www.niu.edu/psychology/britt.

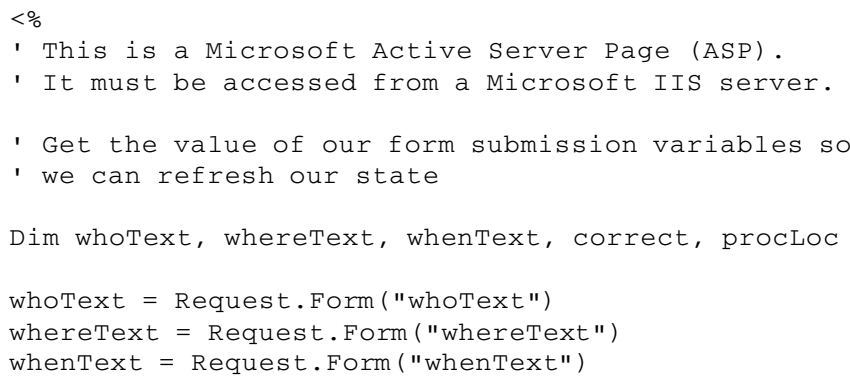




\section{APPENDIX A (Continued)}

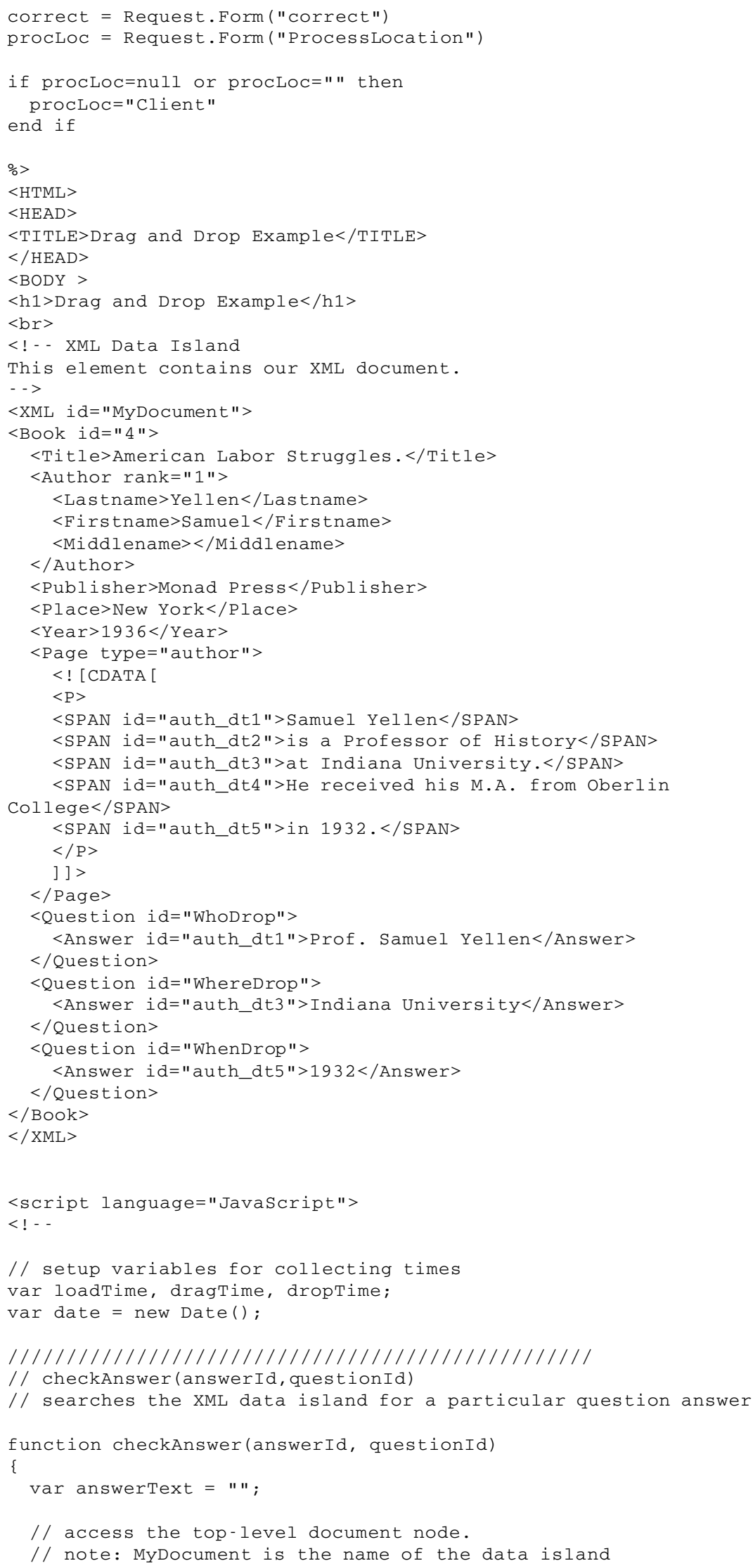


APPENDIX A (Continued)

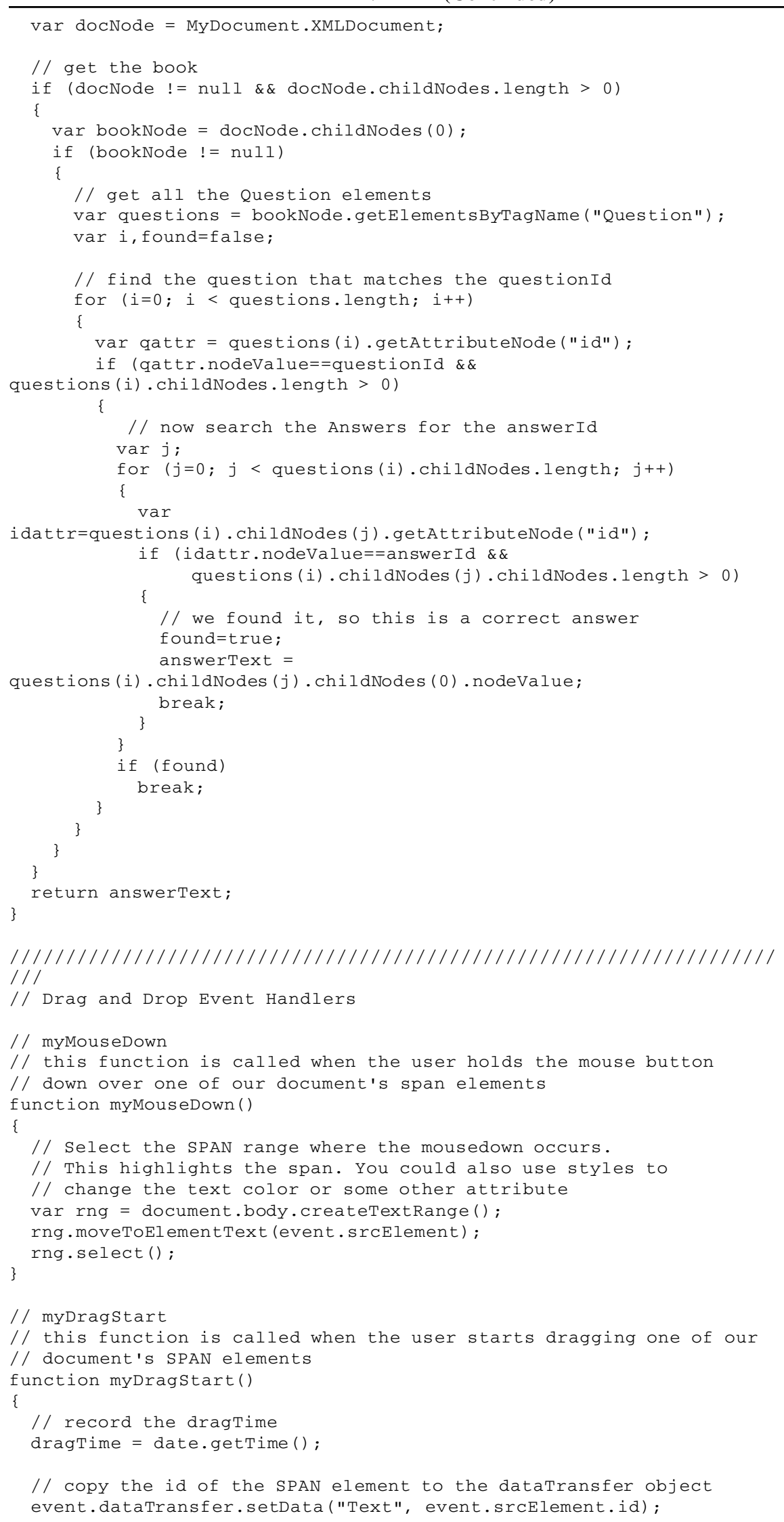


APPENDIX A (Continued)

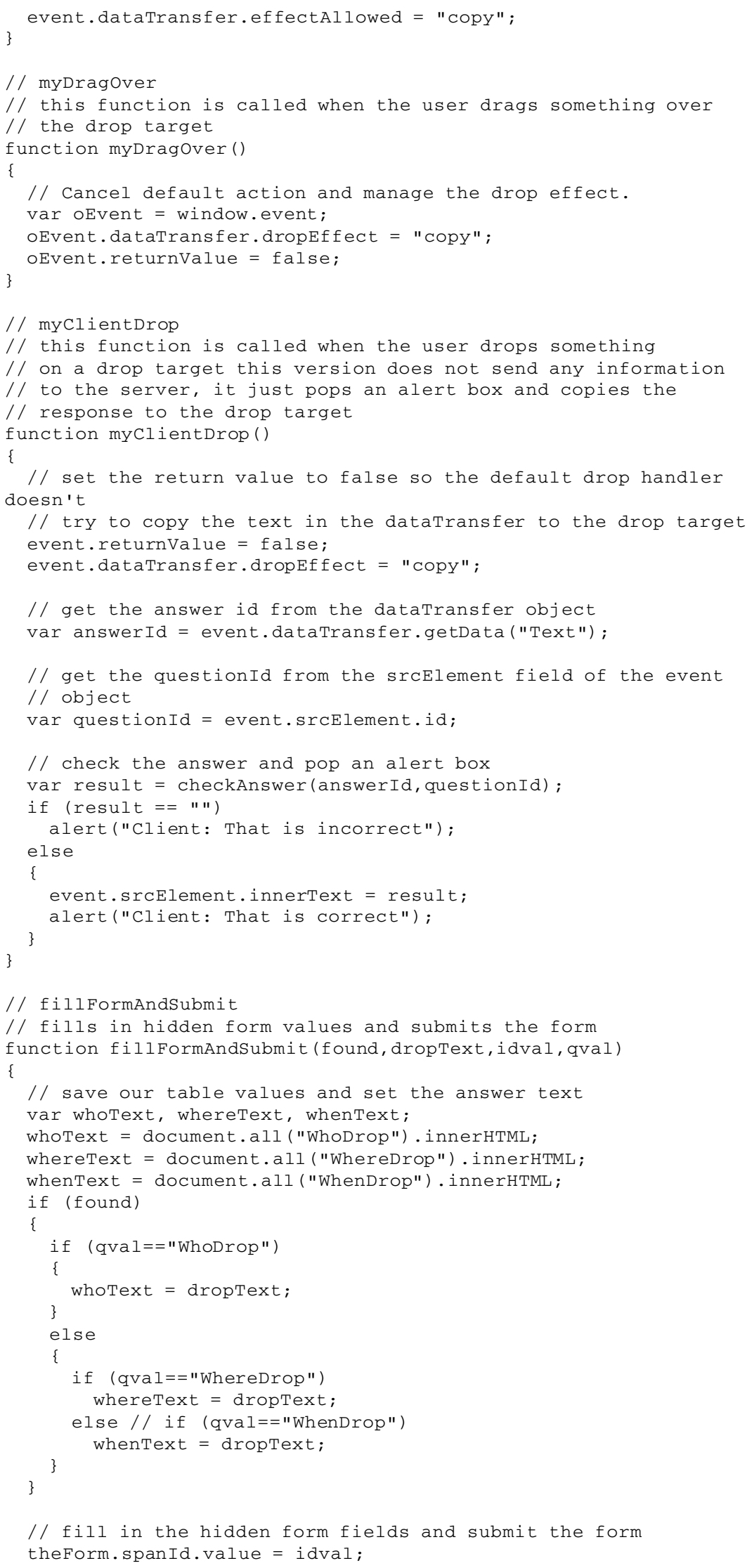


APPENDIX A (Continued)

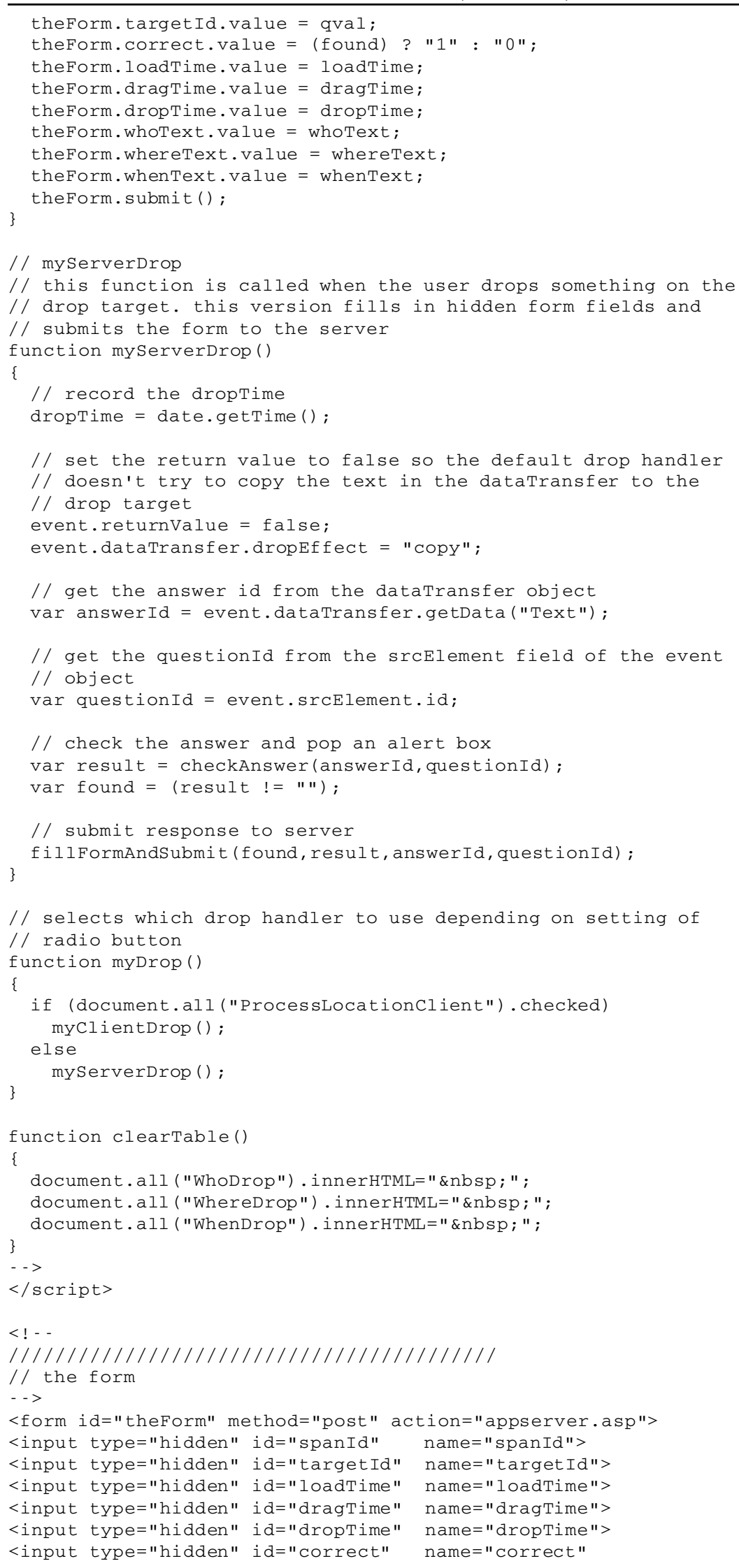


APPENDIX A (Continued)

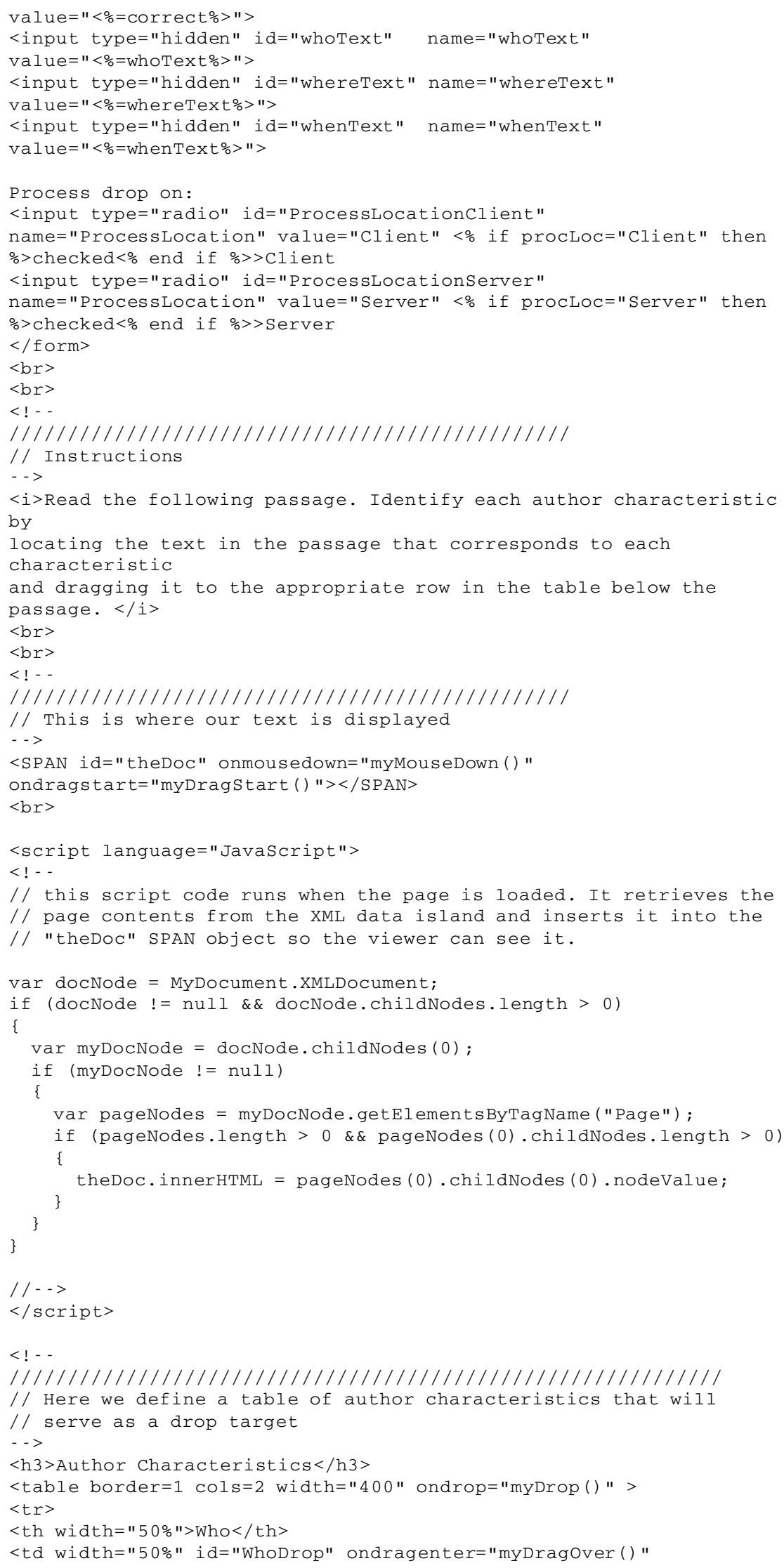


APPENDIX A (Continued)

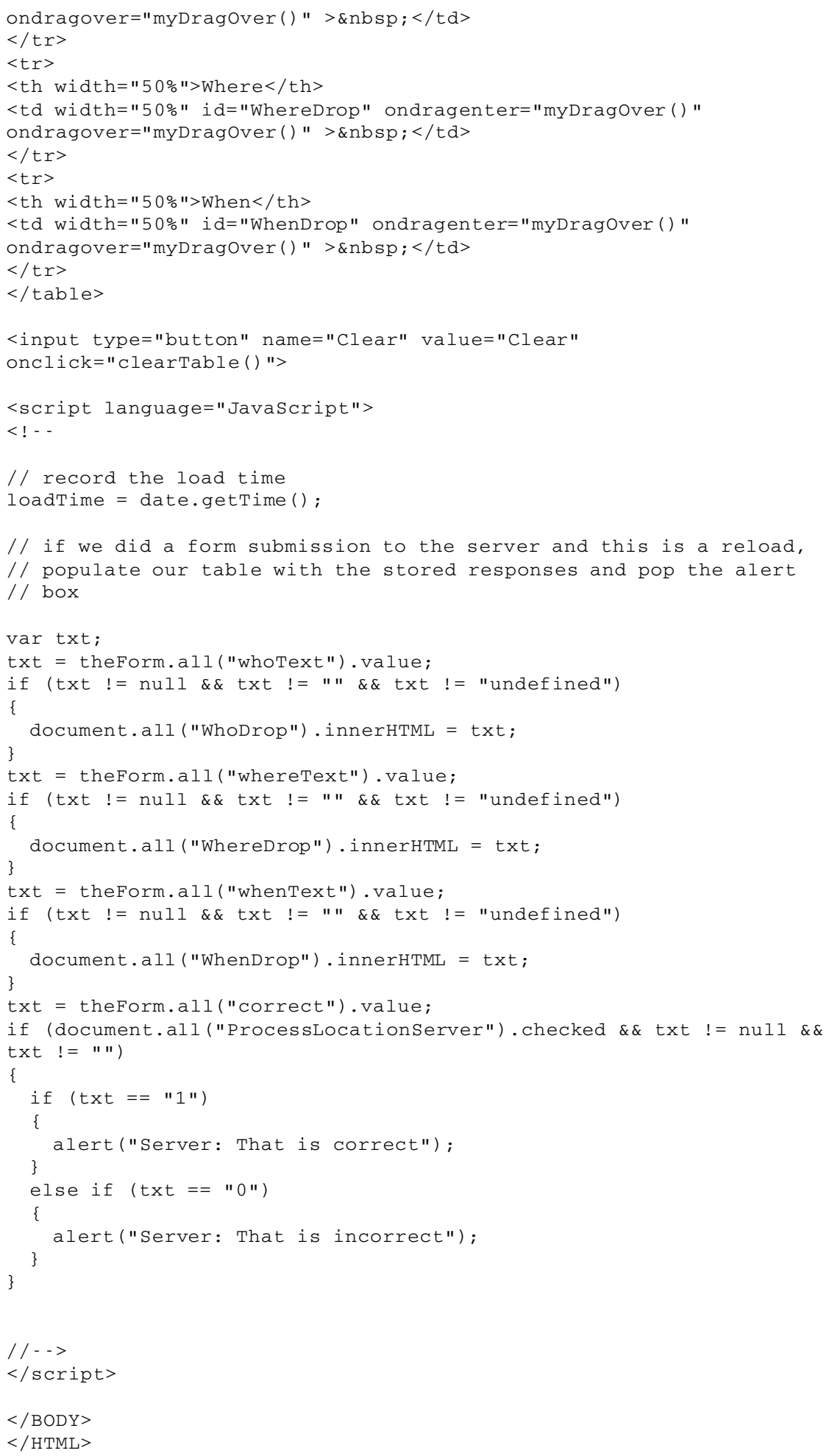




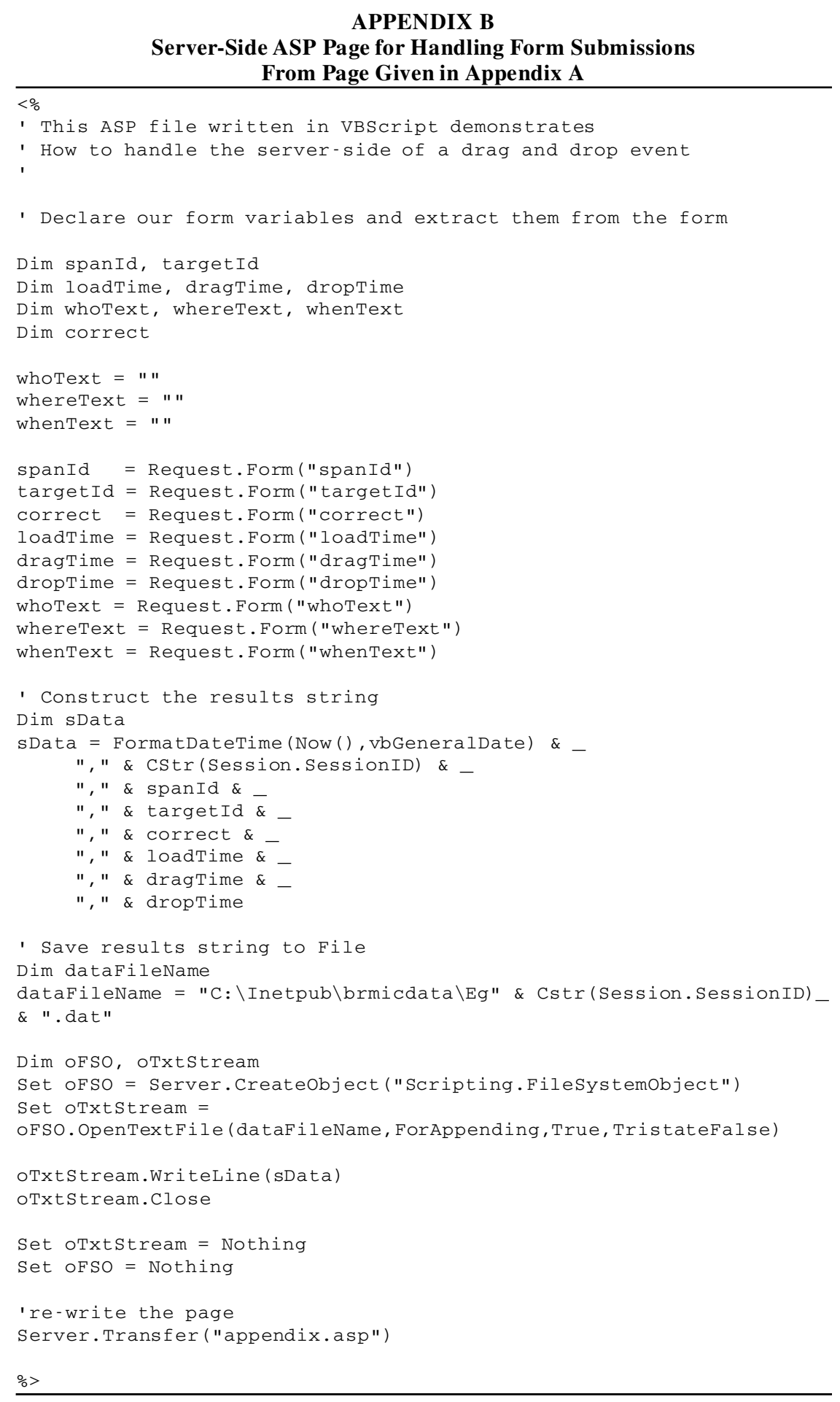

Note—The source code is available at http://www.niu.edu/psychology/britt.

(Manuscript received February 19, 2002;

revision accepted for publication September 6, 2003.) 